\title{
Timely Administration of Thrombolytic Therapy in Acute Ischemic Stroke: An Ethnographic Study
}

\author{
Elmer Catangui ${ }^{1,}$, Elizabeth Baua $^{2}$, Jesus Pizarro ${ }^{3}$ and Adel F. Almutairi ${ }^{4}$ \\ ${ }^{1}$ Director Clinical Nursing, Ministry of National Guard Health Affairs, King Abdulaziz Medical City, Kingdom of Saudi Arabia \\ ${ }^{2}$ Dean, Post Graduate, Academic Supervisor, St Paul University Philippines, Philippines \\ ${ }^{3}$ Academic Supervisor, St Paul University Philippines, Philippines \\ ${ }^{4}$ Academic Supervisor, Chairman, Research Office, King Abdullah International Medical Research Center, Kingdom of Saudi Arabia
}

\section{Abstract}

Background: Thrombolysis treatment is a licensed therapy for acute ischemic stroke within 4.5 hours of stroke onset. The International Guideline sets the door-to-needle (DTN) time is $<60$ minutes. Achieving DTN of 60 minutes or less is tricky and challenging to healthcare organizations due to multifactorial reasons. It is an important study because timely administration of the therapy can improve patient's outcomes and the identified barriers must address appropriately.

Aim: The aim of this study was to explore the views and practices of emergency room (ER) nurses on and "enablers" and "barriers" that affect the DTN time in thrombolysis treatment for acute ischemic stroke using ethnographical research approach.

Method: An ethnographic research method was employed to obtain enriched information and deeper understanding of emergency nurses' practices in delivering timely administration of the thrombolytic therapy in one acute hospital in the Kingdom of Saudi Arabia. Twenty-five samples were participated in the study using a semi-structured interview. The researchers attended every stroke code activation and spent 200 hours during their field work activities.

Results: The enabling practices of timely administration of "tissue plasminogen activator (tPA)" in stroke therapy are mainly dependent on the following success factors: (1) intensive knowledge, (2) skill and (3) positive attitude of nurses toward stroke care; (4) organized system and technology in managing stroke patients in the emergency room, (5) working collaboratively with the team; and (6) well-defined nursing roles in assessing and monitoring stroke patients. On the other hand, the barriers include factors that pertain to patient, staffing, physician, education, resource, and teamwork

Conclusion: A strong leadership and management commitment are vital to ensure that the existing strategies to address the barriers in timely administration of tPA are closely monitored, evaluated, and sustained.

\section{Introduction}

Stroke is a global health issue which is preventable and a treatable health condition. Statistically, stroke affects more than 16 million of people annually [1]. Stroke is also costly. It carries a financial burden not only to stroke survivors' families but also to a country's economy. It is the second leading cause of mortality and the third leading cause of physical disability-adjusted life-years (DALYs) worldwide [2]. Moreover, it is also associated with high mortality rate.

The concept of 'time is brain' has been integrated in the stroke management. Every second counts in stroke management in the emergency unit because if time is wasted, brain tissue is lost. Hence, it is important to act rapidly when stroke strikes because it is an emergency requiring an urgent medical attention. There is an evidencebased treatment given to an eligible stroke patient that can save the brain cells from irreversible damage. It is known as a thrombolysis treatment or a "clot busting" therapy. The word thrombolysis consists of two basic terms-'thrombo' meaning clot and 'lysis' meaning to dissolve or break up. It is a promising treatment for acute ischemic stroke. However, treatment needs to be administered within 4.5 hours of symptom onset [3].

The thrombolytic therapy using alteplase or "tissue plasminogen activator (tPA)" is the only licensed treatment for acute ischemic stroke and it shows favorable outcomes to patients following a stroke [4]. patient's outcome.
In every eight (8) patients who receive the treatment, two (2) of them will improve functionally and can be discharged from the hospital within three (3) days without any physical disability. The thrombolysis therapy offers an opportunity to effectively reduce disability and dependency. However, one (1) in every twenty (20) patients who receives the treatment could potentially have adverse reactions such as bleeding in the brain or elsewhere in the body. The success and benefits of this treatment are time-dependent. The International Guidelines recommend a door-to-needle (DTN) time of $\leq 60$ minutes [5]. The DTN time is defined as the time when a suspected stroke arrives by the door of emergency room (ER) and the time that the thrombolytic therapy is given. The sooner the therapy is given, the higher the chances of patient's recovery from a devastating stroke. Time is of the essence in thrombolytic therapy in acute stroke because timely administration of the treatment can dramatically improve

"Corresponding Author: Dr. Elmer Catangui, Director Clinical Nursing, Ministry of National Guard Health Affairs, King Abdulaziz Medical City, Kingdom of Saudi Arabia; E-mail: catanguiel@ngha.med.sa

Citation: Catangui E, Baua E, Pizarro J, Almutairi AF (2020) Timely Administration of Thrombolytic Therapy in Acute Ischemic Stroke: An Ethnographic Study. Int Nurs Clin Pract 7: 331. doi: https://doi.org/10.15344/2394-4978/2020/331

Copyright: (C) 2020 Catangui et al. This is an open-access article distributed under the terms of the Creative Commons Attribution License, which permits unrestricted use, distribution, and reproduction in any medium, provided the original author and source are credited. 
The need of administering alteplase within 60 minutes is vital. Less than one-third of acute ischemic stroke patients who receive thrombolytic therapy are treated within guideline-recommended DTN times [6]. The emergency healthcare providers (e.g. physicians, imaging, and laboratory technicians) including nurses, being the frontlines in triage, assessment, and management of stroke patients, work collaboratively to address the timely administration of the thrombolytic therapy. Conversely, they are facing multiple challenges in meeting the recommended DTN. Achieving DTN time (60 minutes or less) is tricky, tedious, and ambitious to healthcare organizations due to numerous factors.

Several studies examined the "enabler" factors of achieving DTN time. These factors include introducing a new system of taking acute stroke patients direct from triage to the computerized tomography (CT) imaging [7]; the implementation of a five-simple strategy, namely: call notification, prioritizing patients for CT scan, prioritizing patients for lab analysis, specifying a bed for acute stroke patients, and staff education [8]; the involvement of emergency medicine pharmacists (EMPs) in the administration of tPA by assessing patients for contraindications, preparing and administering tPA [9]; the implementation of a standard operating procedure that defined the targeted standard of care to be provided to all acute stroke patients [10]; and the use of 'code stroke' rapid access protocol [11]. All these strategies and initiatives demonstrate an absolute reduction of DTN time in thrombolytic therapy.

Barriers in timely administration of thrombolysis treatment for acute ischemic stroke include delay in the arrival of ER physician to assess the stroke patient [12]; delay in stroke triage and paging, issues in blood pressure management, fluctuating neurological symptoms uncertainty about diagnosis, delays associated with obtaining consent, and uncertainty about the time of symptom onset [13]; patientrelated reasons account for the longest delays in achieving DTN time, including most prominently the need for emergent medical stabilization including intubation and ventilation, evaluation and treatment of seizure, and difficulty with determining eligibility [14]; and delays in results of laboratory tests could cause unsubstantiated delay of thrombolysis [15].

Some of qualitative studies such as Olson et al. [16], Stecksen et al [17], and Johnson et al. [18] argue that the social, communication, and environmental factors affect the timely administration of thrombolytic therapy. These studies were conducted among physicians, nurses and other disciplines optimizing explorative and phenomenological method. However, there is limited qualitative research that examined the barriers and enablers of timely administration of thrombolytic therapy using an ethnography approach and emergency nurses as participants in the study.

The aim of this study was to explore the views and practices of ER nurses on and "enablers" and "barriers" that affect DTN time in thrombolysis treatment for acute ischemic stroke using ethnographical research approach.

\section{Method}

A qualitative ethnographic research method was used to understand human behavior. It investigated social processes in as normal a way as possible using several linked research methods that emphasize on communication and social interaction. Researchers conducted a semi-structured interview among 25 participants who worked in the emergency room. Twenty-five emergency nurses participated in the semi-structured interview. No one withdrew from the study. They were in the age range of 31 to $50(76 \%)$; majority were female $(76 \%)$; and had been working in the emergency room department ranging from 1 to 10 years (84\%). The participants spent $30-45$ minutes interview with the researchers. Data were analyzed thematically using Braun \& Clark [19]. The research was approved in compliance with Research Ethics Committee of St Paul University Philippines and Ministry of National Guard Health Affairs, King Abdulaziz Medical City Riyadh. It was conducted from October 2019 to January 2020 at the Ministry of National Guard Health Affairs, King Abdulaziz Medical City Riyadh.

\section{Results}

Practices that enabling timely Administration of thrombolytic therapy in Acute Ischemic Stroke

From this study, six themes emerged that underpin "enabling practices" that facilitate timely administration and reduce the DTN time in stroke therapy. These include: (1) nurses' knowledge in stroke thrombolysis; (2) nurses' skill in assessment and history taking; (3) nurses' attitude toward the treatment and response to the stroke code"; (4) patient's full description of the stroke event"; (5) nurses implement organized system and technology; (6) nursing roles are defined, and nurses work with the multidisciplinary team. On the other hand, six themes have been identified as barriers which include factors that pertain to patient, staffing, physician, education, resource, and teamwork.

The study reveals that if stroke patients were descriptive about the event and stated the time of stroke onset, it would be easier for the ER nurses to respond rapidly and be able to notify the physician for stroke code activation. An example report expressed as:

"If patient describes fully what happened and cooperative to the assessment, no doubt, it makes easier for nurses to make decision that this patient is a suspected stroke and I can take him straight to the resuscitation unit". (Nurse participant (NP) 021)

It also posits that the nurse's involvement in attending a stroke code activation and thrombolytic therapy made them feel of "fulfilled" in undertaking the role. They felt that the therapy had favorable outcomes to patients following a stroke. An excerpt described by some participants:

"I had this patient who came in with presenting complaint of rightsided weakness and we gave the tPA within 39 minutes and with my surprise, she started to lift her right arm and recovered quickly, and I was happy on her outcomes. It was an amazing medication. It was a fulfilling and rewarding experience". (NP 014)

The results of interview also revealed that nurses implement and integrate technology in clinical practice and putting system into place which helped to promote a seamless thrombolysis process. It includes (1) stroke thrombolysis pathway, (2) overhead paging system, (3) International Normalized Ratio (INR) point of care device, (4) blue dot in the specimen blood bottle; (5) fast tracking system.

In this study, most participants expressed that they utilized a structured thrombolysis pathway that guide them in the process. Each nurse followed every single step of this pathway (Figure 1). 


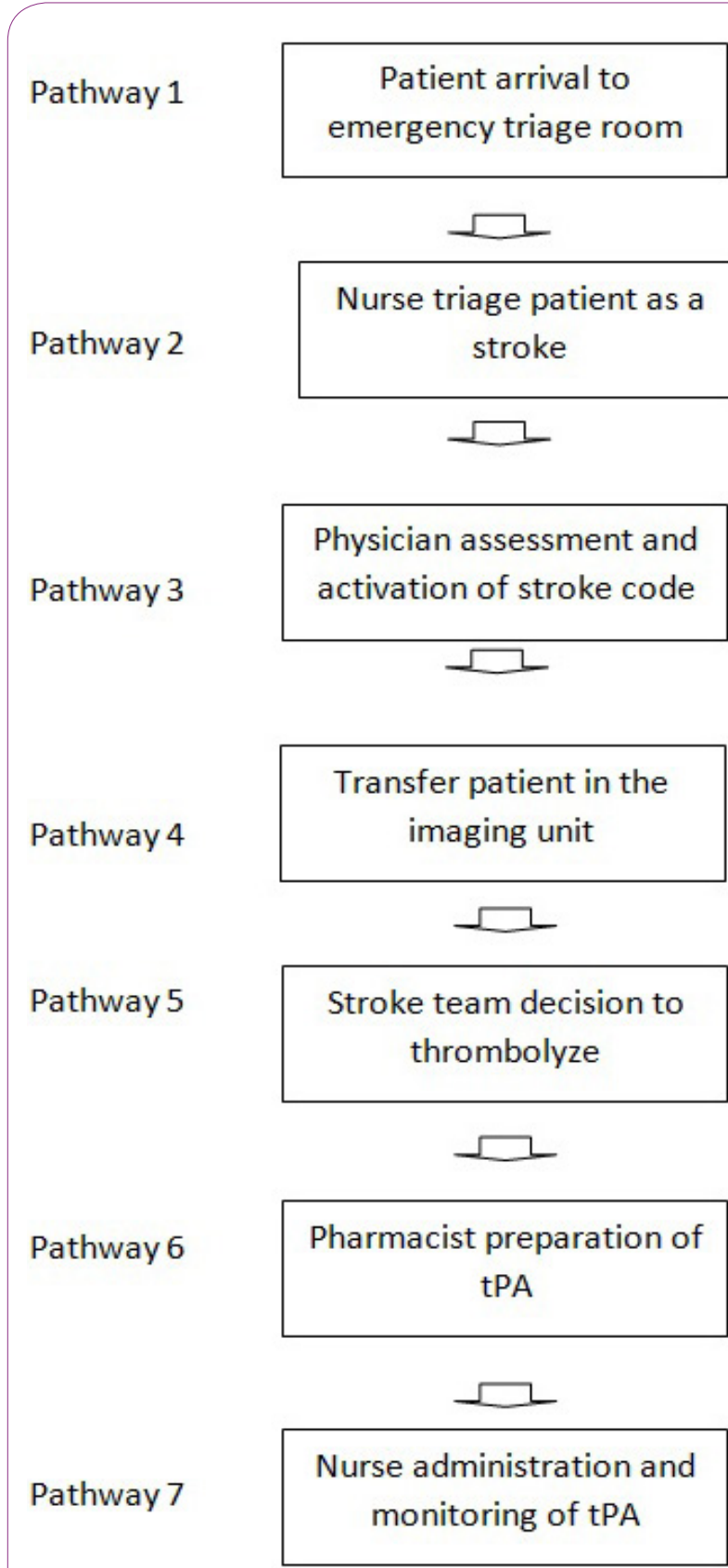

Figure 1: Hospital Stroke Thrombolysis Pathway.

Moreover, another example of anecdotal report that depicts a technology preventing the delay of obtaining blood result especially if patient was on warfarin.

"We have an international normalized ratio (INR) point of care that helps to speed up the result of INR or coagulation which resulted to a reduction of 35 -minute waiting time for the result from the laboratory". (NP 005)

The findings from the interview also summarized definition of nursing roles enabling the thrombolysis treatment for acute ischemic stroke (Figure 2).

\section{Barriers on timely administration of tPA}

Cultural practices were also reported as barriers. The nurses felt that physical assessment was quite impossible if patients wore their abayas (traditional dress) with covered face. It was a challenge for a nurse to determine whether the patient has a facial droop. Furthermore, female patients requested to be examined by the same gender only. Often, some patients had a big family when they arrived in the ER and they considered the eldest member of the family to make the decision if patient could not consent by himself. More often, nurses had difficulty contacting the eldest family member. Obtaining informed consent from patients who are eligible for thrombolytic therapy is crucial. Another factor reported is the language barrier. An example of an excerpt is described below:

"In terms of cultural, I guess it is the language barrier. It is one of the factors. And it is an issue in assessing stroke patients because they speak Arabic and the way nurses ask question, who are not Arabic may misinterpret in a different way." (NP 001)

The emergency department is unpredictable, a hectic, and a busy working environment. The ER nurses worked with multiple tasks and patients who require urgent attention and treatment simultaneously. An example of situation as described below posits the lack of staff.

"Sometimes, we have lots of patients in triage section and we experience lack of nursing staff especially here to assess stroke patients. So, patients need to be waited for long before they will be seen. We cannot attend to stroke patients sometimes due to work overload. Stroke patient is becoming less priority in this case." (NP 004).

Often, stroke is becoming second priority list for assessment. The participants expressed that some physicians in the emergency room did not perceive stroke as an emergency condition and did not believe on the benefit of thrombolytic therapy to patients. This result warrants the need for additional educational updates and training for physicians regarding urgent response to stroke and advocacy of tPA treatment to eligible patients. An example of report is highlighted below:

"Stroke is not marketed it very well. Some physicians do not perceive stroke as an emergency condition like trauma. Some doctors do not believe in the thrombolytic administration and I guess it is an educational need. They themselves don't advocate that the treatment would help patients". (NP 001)

The study ascertains that participants were aware of the capturing the timings for each stroke code activation as an audit project; however, the monthly audit results did not reach the participants working in triage section which stemmed to a lack of information. This finding is evident in one of the reports:

"I am aware that there is an audit going on thrombolysis timings, but no one gets feedback on the result every month." (NP 021)

The participants described their experiences that lead to a communication breakdown. One of them was when physicians did not communicate with nurses about the patient's plan of care. A description of participant's experience is explained as:

"When the stroke team decided to thrombolysis, they put tPA order to the electronic system without informing the emergency nurses, then 


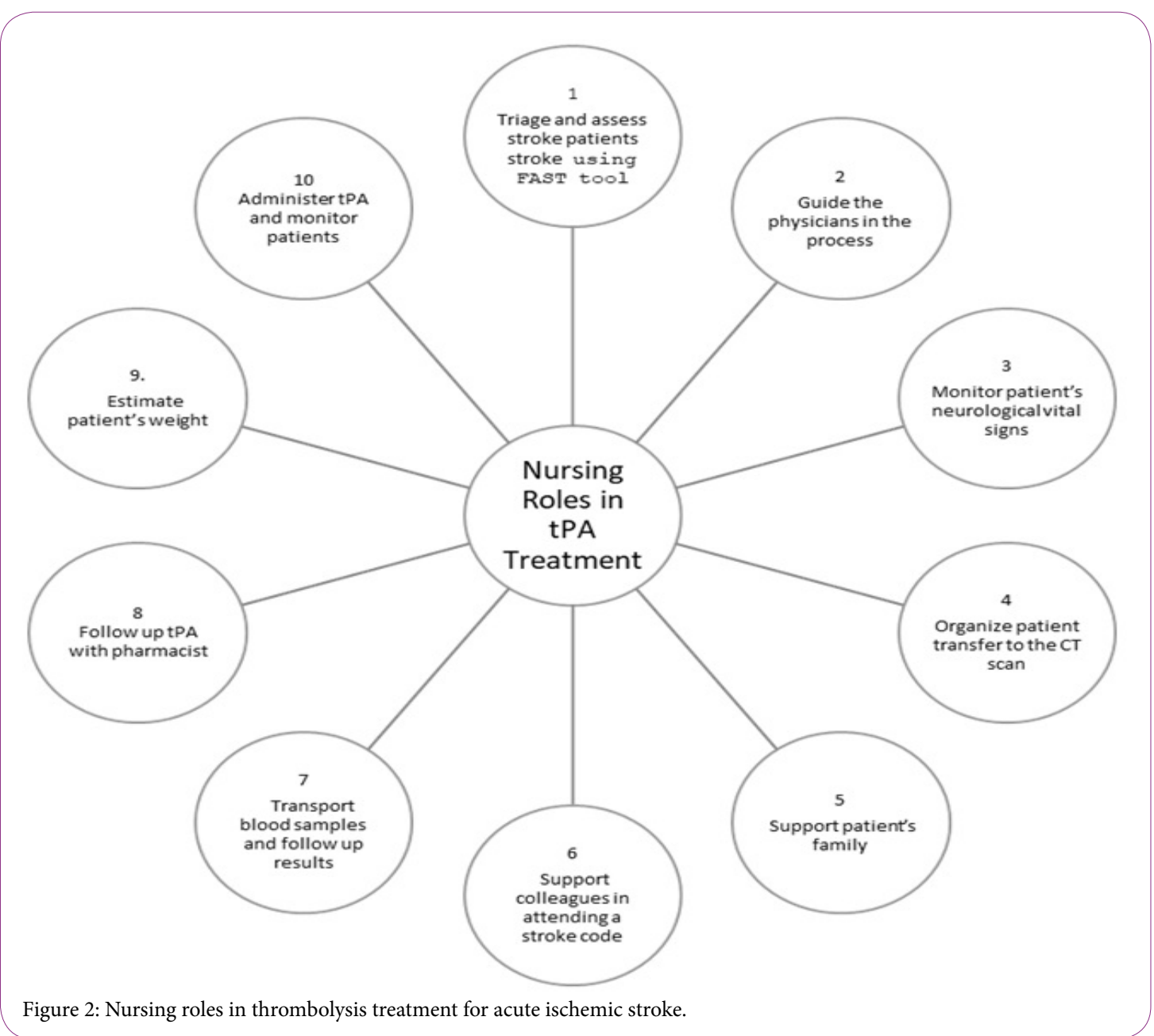

when the stroke team asked us why the tPA has not started yet. We said that we do not know if patient is for thrombolysis. There is a lack of communication here". (NP 022)

\section{Discussion}

Practices that enabling timely Administration of thrombolytic therapy in Acute Ischemic Stroke

The findings suggest the importance of nurses' skill in assessing and taking history from patients or their family to improve the process of patient assessment. It also includes the use of (1) assessment tool, (2) history taking and (3) language. Fawcett \& Rhynass [22] claimed that the focus of history taking involves not only identifying signs and symptoms of illness, but also the individual's experience of illness. Taking a patient history is increasingly being undertaken by nurses as their roles and responsibilities expand. Therefore, it is a way forward to develop this valuable skill of nurses working in advanced practice roles in a clinical setting. Interestingly, some ER nurses are fluent in Arabic language that makes their history taking and patient assessment easier. In practice, it is vital to address language barrier by educating nurses about the country's language or dialect when dealing with patients where English is not their primary language.

In this study, it emerged that nurses' knowledge on thrombolytic therapy stressing on target timelines of the DTN, the integration of "time is brain concept" in practice and application of stroke care guidelines has contributed in achieving DTN. This finding is consistent to the recommendation of Mellon et al. [20] that targeted educational program among hospital staff regarding stroke are required to optimize acute stroke care. Moreover, the literature supports that the nurses' application of stroke knowledge can tremendously influence on early identification of stroke, stroke assessment, thrombolysis process, timing of each pathway, and identifying eligible strokes for the therapy $[21,18]$.

The use of Fast Arm Speech and Time (FAST) as a triage tool helps nurses to recognize stroke symptoms. It is a validated tool to identify stroke symptoms such as facial weakness, arm weakness and speech disturbance. In this study, the ER nurses treated stroke as an emergency condition that requires to be seen immediately by the ER physicians.

Song [23] advocates that a straight forward information from the patient is an essential cue to determine the diagnosis and screen whether a stroke patient is suitable to receive intravenous thrombolysis. However, it is a challenge for nurses if patients suffer from language deficit caused by a stroke.

The literatures embrace the contribution of nurses in facilitating the thrombolysis process, reducing the DTN time, monitoring post thrombolysis patients and improving patient care [24-30]. However, Moran et al. [31,32,29,33] claimed that nursing roles must underpin advanced nursing practice focusing on: (a) advanced assessment and 
triage of stroke patient, (b) taking of clinical history from patient, (c) examining patients using National Institute for Health Stroke Scale (NIHSS) score, (d) obtaining and reviewing imaging, (e) reviewing the indications and contraindications for tPA and discuss the tPA eligibility with the on-call vascular neurologist over the phone, (f) utilizing nurse-led stroke code protocol, (g) ordering and overseeing laboratory and diagnostic test such imaging, blood test, electrocardiogram, and $(\mathrm{h})$ providing 24-hour stroke thrombolysis therapy. These advanced roles were not illuminated in the current study. Hence, capitalizing on nurses in advanced practice in stroke could potentially improve patient's outcomes following a stroke. The contribution of advance nursing practice is a way forward to make a difference to stroke care.

Overall, the use of technology and reconfiguring a system in responding to stroke code activation can reduce the DTN time in stroke therapy. In practical sense, the technology and system work well in speeding up the thrombolysis process that helps in improving the timely administration of thrombolytic therapy [10].

\section{Barriers on timely administration of tPA}

This finding was also supported in the study of Gurav et al. [34,35]. Consequently, Kobayashi et al. [36] recommended increasing staffing level by placing additional on-call staff that takes care only of patients with acute stroke. Stakeholders of the hospital must look at the patient risks resulting from staffing issues in emergency care for stroke and examine the need of more nurse specializing in stroke care.

The physicians' fears of risk of intracranial hemorrhage among other side effects of thrombolytic therapy may play a role in clinical decision to treat patients which affects the DTN [37]. Khathami et al. [38] claimed that the main reasons for emergency physicians who do not recommend tPA use were that they found the said treatment lacking efficacy or effectiveness. The lack of exposure, education and awareness about eligibility could be the contributory factors [39].

Conflict and communication breakdown could delay the timely administration of tPA. Creating a culture of teamwork, communication, collaboration, and coordination in responding to stroke patients in emergency department is essential deliver stroke care and treatment [42].

The DTN audit results serve as a driving force to improve and sustain the timely administration of thrombolytic therapy. Systematic reviews have shown that cycles of audit, with written and verbal feedback can change clinical practice and nominated behaviors by $1 \%$ to $6 \%[40]$.

Our study reports that the lack of resources can also affect the timely administration of tPA. These include the lack of a dedicated stroke bed in the ER, Arabic interpreter, and limited physicians during weekend. It demonstrates that delays in timely administration of tPA were prevalent during weekend and out of hours due to availability of resources. Fang et al. [41] also explained that majority of patients with stroke received thrombolysis during the weekend; therefore, it is imperative to make these resources available during the weekend.

From our study findings, it reflected that patients became indecisive once the risks of the treatment were explained to the patient. All stroke patients must have access to linguistically and culturally appropriate care. The nurses working in the Middle East countries like Saudi must learn the Arabic language that will help them in identifying and addressing patient's needs. Also, it has been suggested that customs, culture, and religion may be factors that delay the accurate diagnosis of patients with suspected acute stroke. In nursing practice, recognizing patient's language and cultural diversity must remain the cornerstones of patient care. Staffing issue has regarded as one of the barriers in this study. In these cases, when the stroke team orders the t-PA therapy, it was considered optimal solution is to automatically notify the stroke nurse through the ordering system.

\section{Conclusion}

The aim of this study was to explore the views and practices of ER nurses on and "enablers" and "barriers" that affect DTN time in thrombolysis treatment for acute ischemic stroke using ethnographical research approach. The "enablers" were nurses' knowledge in stroke thrombolysis; nurses' skill in assessment and history taking; nurses' attitude toward the treatment and response to the stroke code"; patient's full description of the stroke event"; nurses implement organized system and technology; nursing roles are defined, and nurses work with the multidisciplinary team. On the other hand, the "barriers" were factors that pertain to patient, staffing, physician, education, resource, and teamwork. These barriers were foreseeable, preventable, and manageable. These must be addressed by improving coordination and collaborative team work, developing competencies of nurses, increasing staffing during weekend and appropriate resources.

\section{Implications of this study to nursing practice and education}

Thrombolysis in acute ischemic stroke is a time-dependent therapy and it needs to be given within the target DTN time. Nurses have facilitating, supporting, leading, and advocating roles in thrombolysis therapy for acute ischemic stroke. The identified barriers in timely administration of tPA can be addressed by increasing public awareness of stroke symptoms and urgent seeking medical attention, intensifying education for nurses, enhancing competencies of nurses in peripheral insertion, improving communication and coordination between physicians and nurses, reviewing the thrombolysis pathway to address who will activate the stroke code, allocation of dedicated bed for stroke patient in the emergency room unit, increasing staffing both for nursing and physician workforce and improving proper documentation. Developing nursing practice in the provision of emergency care for stroke patients can be enhanced by intensifying a continuous education both basic and advanced knowledge in stroke assessment and thrombolysis care.

\section{Competing Interests}

The authors declare that they have no competing interests.

\section{References}

1. World Health Organization (2018) Cerebrovascular Accident- Stroke. WHO: Geneva.

2. Hankey G (2013) The global and regional burden of stroke. Lancet Glob Health 1: 239-240.

3. Lansberg MG, Bluhmki E, Thijs VN (2009) Efficacy and safety of tissue plasminogen activator 3 to 4.5 hours after acute ischemic stroke: a metaanalysis. Stroke 40: 2438-2441.

4. Bansall S, Sangha KS, Khatri P (2013) Drug treatment of acute ischemic stroke. Am J Cardiovasc Drugs. 
Citation: Catangui E, Baua E, Pizarro J, Almutairi AF (2020) Timely Administration of Thrombolytic Therapy in Acute Ischemic Stroke: An Ethnographic Study. Int J Nurs Clin Pract 7: 331. doi: https://doi.org/10.15344/2394-4978/2020/331

Page 6 of 6

5. The American Heart and Stroke Association (2010) Target Stroke. Time lost mean brain lost. Target: Stroke Campaign.

6. Fonarow GC, Smith EE, Saver JL, Reeves MJ, Hernandez AF, et al. (2011) Improving Door-to-Needle Times in Acute Ischemic Stroke the Design and Rationale for the American Heart Association/American Stroke Association's Target: Stroke Initiative. Stroke 42: 2983-2989.

7. Panezai S, Chukwuneke F, Arango A, Jaskiran B, Daniel J, et al. (2016) Overcoming barriers to reduce door to needle time in acute ischemic stroke patients: Field to CT. Neurology 86: 6 .

8. Sadeghi-Hokmabadi E, Taheraghdam A, Hashmemilar M, Ridkhtegar R Mehrvar K, et al. (2016) Simple-hospital interventions to reduce door to CT time in acute stroke.

9. Jacobyt JS, Draper HM, Dumkow LE, Farooq MU, DeYoung GR, et al. (2018) Emergency medicine pharmacist impact on door-to-needle time in patient with acute ischemic stroke. Neurohospitalist 8: 60-65.

10. Van Schaik SM, der Veen BV, Van den Berg-Vos RM, Weinstein HC Bosboom WMJ (2014) Achieving a Door-to-Needle Time of 25 minutes in thrombolysis for acute ischemic stroke: A quality improvement project. Stroke Cerebrovascular Disease 23: 2900-2906.

11. Tai YJ, Weir L, Hand P, Davis S, Yan B, et al. (2012) Does a 'code stroke' rapid access protocol decrease door-to-needle time for thrombolysis? Intern Med J 42: 1316-1324.

12. Fernandes D, Umasankar U (2016). BMJ Quality Improvement Report.

13. Mowla A, Doyle J, Lail NS, Rajabzadeh-Oghaz H, Deline C, et al. (2017) Delays in door-to-needle time for acute ischemic stroke in the emergency department: A comprehensive stroke center experience. J Neurol Sci 376: 102-105.

14. Choi PMC, Desai JA, Kashyap D, Stephenson C, Kamal N, et al. (2016) Are all stroke patients eligible for fast alteplase treatment? An analysis of unavoidable delays academic. Acad Emerg Med 23: 393-399.

15. Breuer L, Huttner HB, Kiphuth IC, Ringwald J, Hilz MJ, et al. (2013) Waiting for platelet counts causes unsubstantiated delay of thrombolysis therapy. Eur Neurol 69: 317-320.

16. Olson DM, Constable M, Britz GW, Lin CB, Zimmer LO, et al. (2011) A qualitative assessment of practices associated with shorter door-to-needle time for thrombolytic therapy in acute ischemic stroke. J Neurosci Nurs 43 : 329-335.

17. Stecksen A, Lundman B, Eriksson M, Glader E (2014) Implementing thrombolytic guidelines in stroke care. Qual Health Res 24: 412-419.

18. Johnson M, Cohn J, Bakas T (2011) Emergency department nurses' perceived barriers and facilitators to caring for stroke patients. J Neurosci Nurs.43: 238-243.

19. Braun V, Clarke V (2006) Using thematic analysis in psychology. Qual Res Psych 3: 77-101.

20. Mellon L, Hasan H, Lee S, Williams D, Hickey A, et al. (2015) Knowledge of thrombolytic therapy amongs hospital staff: preliminary results and treatment implications. Stroke 46: 3551-3553.

21. Barbour V, Thakore S (2017) Improving door to CT scanner times for potential stroke thrombolysis candidates- the Emergency Department's role. BMJ Qual Improv Rep 6: u211470.w4623.

22. Fawcett T, Rhynas S (2012) Taking a patient history: the role of the nurse. Nursing Standard 26: 41-46.

23. Song S (2013) Hyperacute management of ischemic stroke. Semin Neurol 33: 427-435.

24. Catangui EJ, Slark J (2012) A thrombolysis pathway for patients following acute ischemic stroke. Nurs Stand 26: 35-42.

25. English $\mathrm{V}$ (2019) Time is brain: The role of a nurse in acute ischemic stroke Nursing Critical Care 11: 43-46.

26. Kummarg U, Sindhu S, Muengtaweepongsa S (2018) The early outcomes of nurse case management in patients with acute ischemic stroke treated with intravenous recombinant tissue plasminogen activator: A randomized controlled trial. Neurol Res Int 2018: 1717843.

27. Fitzpatrick M, Birns J (2004) Thrombolysis for acute ischemic stroke and the role of a nurse. Br J Nurs 13: $1170-1174$

28. Barclay J, Jones D (2018) Stroke 4: Immediate treatment of acute stroke and TIA. Nursing Times 114: 51-54.
29. Zhou Y, Xu Z, Liao J, Feng F, Men L, et al. (2016). New Standardized Nursing Cooperation Workflow to reduce stroke thrombolysis delays in patients with acute ischemic stroke. Neuropsychiatr Dis Treat 13: 1215-1220.

30. Liu Z, Zhao Y, Liu D, Guo ZN, Jin H, et al. (2018) Effects of nursing quality improvement on thrombolytic therapy for acute ischemic stroke. Front Neurol 9: 1025.

31. Moran JL, Nakagawa K, Asai SM, Koenig MA, et al (2016) $24 / 7$ Neurocritical care nurse practitioner coverage reduced door-to-needle time in stroke patients treated with tissue plasminogen activator. J Stroke Cerebrovasc Dis 25: 1148-1152.

32. Catangui EJ, Roberts CJ (2014) The Lived Experiences of Nurses in one Hyperacute Stroke Unit. Br J Nurs 23: 143-148.

33. Alexandrov AWT, Albright KC, DiBiase S (2011) Post graduate academic neurovascular fellowship for advance practice nurses and physician assistants significantly increases tPA treatment rates. Stroke 42: e206.

34. Gurav SK, Zirpe KG, Wadia RS, Pathak MK, Deshmukh AM, et al. (2015) Problems and limitations in thrombolysis of acute stroke patients at a tertiary care center. Indian J Crit Care Med 19: 256-269.

35. Hargis M, Shah JN, Mazabob J, Rao CV, Suarez JI, et al. (2015) Barriers to administering intravenous tissue plasminogen activator (tPA) for acute ischemic stroke in the emergency department: a cross-sectional survey of stroke centers. Clin Neurol Neurosurg 135: 79-84.

36. Kobayashi A, Marta S, Tomasz L, Anna C (2007) Lack of experience of intravenous thrombolysis for acute ischemic stroke does not influence the proportion of patients treated. Emerg Med J 24 96-99.

37. Asdaghi N, Wang K, Cliliberti-Vargas M, Gutierrez C, Koch S, et al. (2018). Predictors of thrombolysis administration in mild stroke. Stroke. 49: 638645.

38. Al Khathaami A, Aloraini $\mathrm{H}$, Almudlej $\mathrm{S}$, AL Issa H, Elshammaa N, et al. (2018) Knowledge and attitudes of Saudi emergency physicians toward t-pa use in stroke. Neurology Research International.

39. Williams JM, Jude MR, Levi CR (2013) Recombinant tissue plasminogen activator (rt-PA) utilization by rural clinicians in acute ischemic stroke: A survey of barriers and enabler. Aust J Rural Health 21: 262-267.

40. Ivers N, Jamtvedt G, Flottorp S, Young JM, Odgaard-Jensen J, et al. (2012) Audit and feedback: effects on professional practice and healthcare outcomes. Cochrane Database Syst Rev.

41. Fang J, Saposnik G, Silver FL, Karpral MK (2010) Investigators of the Registe of the Canadian Stroke Network, Association between weekend hospital presentation and stroke fatality. Neurology 75: 1589-1596.

42. Fuhrman S, Lazaro M, (2019) Stroke Door-to Needle Target of 30 minutes or Less: A single center experience. Stroke.

This article was originally published in a special issue:

Psychiatric and Mental Health Nursing

Handled by Editor(s):

Prof. Tetsuya Tanioka

Department of Nursing Outcome Management

Tokushima University

Japan 